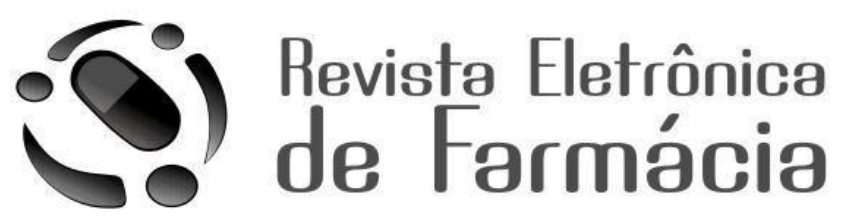

\title{
A IMPORTÂNCIA ECONÔMICA DA COMPRA CENTRALIZADA DOS INIBIDORES DO FATOR DE NECROSE TUMORAL-ALFA EM PERNAMBUCO
}

THE ECONOMIC IMPORTANCE ABOUT CENTRALIZED PURCHASING OF TUMOR NECROSIS FACTOR-ALPHA INHIBITORS IN PERNAMBUCO

LA IMPORTANCIA ECONÓMICA DE LA CENTRALIZADA ADQUISICIÓN DE INHIBIDORES DEL FACTOR DE NECROSIS TUMORAL ALFA EN PERNAMBUCO

Fernando Zanghelini, André Santos da Silva, José de Arimatea Rocha Filho

Recebido em 01/04/2014, Aceito em 25/04/2014.

RESUMO: Analisou-se o impacto nos custos da aquisição centralizada de medicamentos de alta complexidade para o tratamento da artrite reumatoide (AR) na Secretaria de Saúde de Pernambuco. Realizou-se um estudo transversal, comparativo de custo, com medicamentos adquiridos em licitações de 2010 (compra descentralizada) e 2012 (compra centralizada). O custo anual atualizado, com a compra descentralizada dos inibidores de Fator de Necrose Tumoral-Alfa (TNF-a) chegaria ao estimado de $\mathrm{R} \$ 29.640 .229,68$, frente a $\mathrm{R} \$ 15.339 .978,00 \mathrm{da}$ compra centralizada, perfazendo uma economia de 48,2\%. Este resultado demonstrou que a compra centralizada pelo Ministério da Saúde possibilitou economia de recursos, sugerindo uma importante ferramenta para a melhoria da gestão da Assistência Farmacêutica nos estados.

PALAVRAS-CHAVE: Assistência Farmacêutica. Políticas Públicas. Economia da Saúde. Farmacoeconomia. 
ABSTRACT: We analyzed the cost impact of centralized procurement of drugs of high complexity for the treatment of rheumatoid arthritis (RA) in the Health Department of Pernambuco. We conducted a cross-sectional study comparing cost with drugs purchased at bidding from 2010 (not centralized purchasing) and 2012 (centralized purchasing). The cost updated, with decentralized purchasing of inhibitors of Tumor Necrosis Factor-Alpha (TNF-a) arrive an estimated R\$ $29.640 .229,68$ compared to $\mathrm{R} \$ 15.339 .978,00$ of centralized purchasing, making a savings of $48,2 \%$. This result showed that centralized purchasing by the Ministry of Health allowed saving resources, suggesting an important tool for improving the management of Pharmaceutical Care in the states.

KEYWORDS: Pharmaceutical Services. Public Policies. Health Economics. Economics, Pharmaceutical.

RESUMEN: Se analizó el impacto en los costos de adquisición centralizada de medicamentos de alta complejidad para el tratamiento de la artritis reumatoide (AR) en el Departamento de Salud de Pernambuco. Se realizó un estudio transversal, comparación de costos con los medicamentos adquiridos en 2010 subastas (compra descentralizada) y 2012 (compra centralizada). El costo anual actualizado, com la compra descentralizada de los inhibidores de factor de necrosis tumoral alfa (TNF-a) para llegar a un valor estimado de $\mathrm{R} \$ 29,640,229.68$, frente a los $R \$ 15,339,978.00$ de compras centralizadas, lo que hace un ahorro de $48,2 \%$. Este resultado demuestra que la compra centralizada por el Ministerio de Salud ha permitido el ahorro de recursos, lo que sugiere una importante herramienta para mejorar la gestión de la asistencia farmacéutica en los estados.

PALABRAS-CLAVE: Servicios Farmacéuticos. Políticas Públicas. Economía de la Salud. Economía Farmacéutica.

\section{INTRODUÇÃO}

De acordo com a Política Nacional de Medicamento (PNM) de 1998, a Assistência Farmacêutica deve promover o uso racional de medicamentos, otimizar com eficácia o sistema de distribuição, desenvolver iniciativas que possibilitem a redução nos preços dos produtos, de forma que viabilize o acesso da população aos medicamentos. Ainda de acordo com a PNM, a Assistência Farmacêutica no SUS engloba as atividades de: seleção, programação, aquisição, armazenamento, distribuição, dispensação, controle de qualidade e utilização. Desta forma, de acordo com os critérios epidemiológicos locais, a Assistência Farmacêutica visa a permanente disponibilidade de produtos terapêuticos segundo as necessidades da população ${ }^{1}$.

Estudos realizados em diversos países demonstraram que nos últimos anos houve um aumento significativo nos gastos com aquisição de medicamentos em relação aos gastos totais em saúde. Estudo realizado por Kondro (2007), no Canadá, estimou um crescimento de $6 \%$ em 2006, chegando a US\$ 25 bilhões $^{2}$. Nos Estados Unidos o governo federal estima que os gastos com medicamentos continuem a crescer 
nos próximos anos, passando de US\$ 184 bilhões de 2003 para cerca de US\$ 519 bilhões em $2013^{3}$.

No Brasil, segundo dados do Ministério da Saúde, os gastos com aquisição de medicamentos no período de 2002 e 2006, aumentaram em quase $124 \%$, enquanto os gastos totais em saúde aumentaram em $9,6 \%{ }^{4}$.

Para regulamentar 0 financiamento e a transferência dos recursos para compra de medicamentos da Assistência Farmacêutica (AF) o Ministério da Saúde (MS) instituiu a Portaria GM/MS no 204, de 29 de janeiro de 2007. Nela, a forma de financiamento ficou dividida em três blocos: dos medicamentos básicos, dos medicamentos estratégicos e dos medicamentos de dispensação excepcional ${ }^{5}$.

Recentemente, para ampliar o acesso da população aos serviços de saúde de média e alta complexidade, incluindo acesso aos medicamentos de alto custo no âmbito do SUS, o MS, através da Portaria no 2.891, de 26 de novembro de 2009, atualizou o financiamento dos componentes da AF aprovando os Componentes: Básico, Estratégico e Especializado, este ultimo substituiu o Componente de Medicamentos de Dispensação Excepcional ${ }^{6}$.
Os medicamentos que constituem as linhas de cuidado para doenças contempladas na alta complexidade estão no Componente Especializado de Assistência Farmacêutica (CEAF), e dividem-se em três grupos com características, responsabilidades e formas de organização distintas. O Grupo 1, é constituído por medicamentos que representam 0 de maior custo financeiro para o Componente. Seu financiamento está sob a responsabilidade exclusiva da União. O Grupo 2 é constituído por medicamentos, cuja responsabilidade de financiamento é das Secretárias Estaduais da Saúde (SES). Por fim o Grupo 3 é constituído por medicamentos, cuja a responsabilidade pelo financiamento é tripartite ${ }^{6}$.

Em 2003, os recursos gastos pelo Componente de Medicamentos de Dispensação Excepcionais foram na ordem de R\$ 516 milhões, já em 2006 os gastos passaram para $\mathrm{R} \$ 1,3$ bilhões, perfazendo um aumento de $159 \%$ no período, tornando-se um dos grandes componentes no aumento dos gastos com medicamentos do $\mathrm{MS}^{7}$.

Dentre as diversas doenças atendidas pelo CEAF, destacamos a artrite reumatoide, cujas linhas de cuidado estão definidas em Protocolos 
Clínicos e Diretrizes Terapêuticas (PCDT) publicadas pelo $\mathrm{MS}^{8}$.

A Artrite Reumatoide (AR) é uma doença inflamatória crônica, de caráter autoimune, caracterizada por dor e edema, que acomete predominantemente as articulações periféricas de forma progressiva e em longo prazo esta associada à morbidade e mortalidade ${ }^{9}$. Estima-se que a prevalência da $A R$ nos países desenvolvidos varie entre $0,3 \%$ e $1 \%$ da população ${ }^{10}$. Embora a doença possa ter início em qualquer idade, uma maior incidência é apresentada entre as mulheres adultas com idade superior aos 40 anos $^{11}$. Com objetivo de estimar a prevalência da AR no Brasil um estudo realizado por Senna e colaboradores (2004) observaram uma prevalência de $0,46 \%$ da população, com predominância entre as mulheres ${ }^{12}$. Corroborando com os resultados apresentados em países desenvolvidos.

Um estudo realizado por Pincus (1995) com o objetivo de estimar as consequências médicas e econômicas da $A R$, demonstrou que, mais de $50 \%$ dos pacientes com AR e idade inferior à 65 anos, que se encontravam no mercado de trabalho, receberam auxílio doença referente a incapacidade laboral durante o estágio inicial da doença ${ }^{13}$.

A interrupção das atividades profissionais é apenas uma parte do quadro da deficiência resultante da AR. Quando não tratada de forma adequada em tempo hábil, pode ocasionar significativo impacto socioeconômico e sob a qualidade de vida do paciente ${ }^{14}$.

O tratamento farmacológico adequado para estabilizar os sintomas da AR incluem analgésicos opióides e não opióides, anti-inflamatórios não esteróides (AINES), corticosteróides, drogas modificadoras da doença reumática (DMARDs), tal como difosfato de cloroquina e sulfato de hidroxicloroquina, sulfassalazina, metotrexato de sódio, leflunomida e ciclosporina; bem como os DMARDs biológicos, que incluem os inibidores do Fator de Necrose Tumoral Alfa (anti-TNF-a): $\quad$ Adalimumabe $^{15}$, Etanercept ${ }^{16}$, Infliximabe ${ }^{17}$, anticorpos anticelulas B Rituximabe ${ }^{18}$ e o primeiro inibidor da Interleucina - 6 (IL-6) Tocilizumabe ${ }^{19}$.

Objetivando, dentre outros, obter o benefício econômico da centralização frente às condições de mercado e os investimentos estratégicos do governo, o MS juntamente com as Secretárias Estaduais de Saúde (SES) e o Distrito Federal, através da Portaria 2.891 de 2009, pactuaram a aquisição centralizada dos medicamentos pertencentes aos Grupos $1 \mathrm{~B}$ e 2 do $\mathrm{CEAF}^{6}$. 
ZANGHELINE, F.; SILVA, A. S.; FILHO, J. A. R.

REF-ISSN1808-0804 Vol.XI (2),15-24, 2014.

Neste contexto, o presente estudo analisou e comparou os custos totais da aquisição centralizada pelo MS dos anti-TNF-a (adalimumabe, etanercepte e infliximabe), utilizados no tratamento da artrite reumatoide $(A R)$ frente à aquisição descentralizada realizada pelo CEAF da Secretaria Estadual de Saúde de Pernambuco (SES/PE).

\section{MÉTODOS}

Realizou-se um estudo observacional, transversal no Componente Especializado de Assistência Farmacêutica (CEAF) da Secretaria Estadual de Saúde de Pernambuco (SES/PE), situada no Bairro da Boa Vista, município do Recife.

Foram analisados os custos das aquisições dos medicamentos antiTNF-a utilizados no tratamento de 525 pacientes com $A R$, cadastrados no CEAF da SES/PE. A coleta de dados seguiu-se através do Sistema Informatizado de Gerenciamento e Acompanhamento de Medicamento do CEAF (SISMEDEX), com mês e ano base em maio de 2012.

Para análise dos dados, foram considerados aqueles pacientes que apresentaram as seguintes Classificações Internacional da Doença (CID) diagnosticada: M05.0 Síndrome de Felty; M05.1 - Doença reumatoide do pulmão; M05.2 -
Vasculite reumatoide; M05.3 - Artrite reumatoide com comprometimento de outros órgãos ou sistemas; M05.8 Outras artrites reumatoides soropositivas; M06.0 - Artrite reumatoide soronegativas; M06.8 Outras artrites reumatoides e M08.0 Artrite reumatoide juvenil.

As informações com dados absolutos referentes aos valores unitários de cada anti-TNF-a adquiridos no processo licitatório de 2010, ano em que o processo de compra era descentralizado, e de 2012, com processo de compra centralizado totalmente implantado, foram obtidos pela Superintendência de Assistência Farmacêutica de Pernambuco (SAF) fornecida pela Comissão Permanente de Licitação de Medicamentos (CPLM) da SES/PE.

Para cálculo dos gastos mensais com cada anti-TNF- $a$, baseou-se nas dosagens usuais recomendadas no Brasil, atraves do Protocolo Clínico e Diretrizes Terapêuticas (PCDT) para tratamento da $A R$, e incluídas nos estudos de metanálise ${ }^{8}$ : adalimumabe $20 \mathrm{mg}$ por via subcutânea uma vez por semana ou $40 \mathrm{mg}$ uma vez a cada 15 dias; etanercepte $25 \mathrm{mg}$ por via subcutânea duas vezes por semana ou $50 \mathrm{mg}$ uma vez por semana; infliximabe $3 \mathrm{mg} / \mathrm{kgpor}$ via endovenosa nas semanas $0,2,6$ e depois a cada oito semanas. A dose de infliximabe foi 
calculada baseada em paciente de $70 \mathrm{~kg}$.

Obejtivando atualizar os valores unitários de cada anti-TNF-a adquiridos no processo licitatório de 2010 (processo de compra descentralizada) para o ano base da avaliação (2012), foram utilizados o Índice Nacional de Preço ao Consumidor (INPC). A atualização de preço dos anti-TNF-a adquiridos no processo de compra descentralizada (2010) foi realizada pela variação do INPC contabilizada entre o período de janeiro de 2010 à maio de 2012, onde aplicou-se a taxa de juros do período sobre os valores base de 2010. Após atualizar os valores unitários de cada
anti-TNF-a, foram comparadas as diferenças em reais e o impacto mensal nos gastos com a aquisição centralizada de cada item.

\section{RESULTADOS}

$\mathrm{Na}$ aquisição de medicamentos anti-TNF-a para o tratamento de 525 pacientes com AR adquiridos através do processo de compra centralizada pelo MS. Verificou-se que o infliximabe e (IFX) apresentou a maior redução mensal dos valores percentuais $(51,4 \%)$, seguido do etanercepte (ETA) e adalimumabe (ADA) com $50,5 \%$ e $43,7 \%$ respectivamente, conforme apresentado na Tabela 1.

Tabela 1- Distribuição dos custos de aquisição de medicamentos anti-TNF-a e sua variação econômica anual, segundo os tipos de compra descentralizada e centralizada para 525 pacientes com AR, no CEAF da SES/PE em 2012.

\begin{tabular}{|c|c|c|c|c|}
\hline & ADA, 40mg & ETA, $25 \mathrm{mg}$ & ETA, 50mg & IFX, $10 \mathrm{mg}$ \\
\hline Numero de pacientes cadastrados & 195 & 29 & 241 & 60 \\
\hline $\begin{array}{l}\text { Quantidade utilizada por paciente ano } \\
\text { (ampola ou seringa) }\end{array}$ & 24 & 96 & 48 & 24 \\
\hline $\begin{array}{l}\text { Preço Unitário (compra descentralizada, } \\
\text { 2010)* }\end{array}$ & $R \$ 1.900,24$ & $R \$ 546,63$ & $\mathrm{R} \$ 1.093,27$ & $R \$ 2.084,86$ \\
\hline Preço corrigido pelo INPC** $(+13,72 \%)$ & $R \$ 2.160,97$ & $\mathrm{R} \$ 621,63$ & $\mathrm{R} \$ 1.243,27$ & $R \$ 2.370,92$ \\
\hline $\begin{array}{l}\text { Preço Unitário (compra centralizada, } \\
2012 \text { )* }\end{array}$ & $\mathrm{R} \$ 1.217,13$ & $\mathrm{R} \$ 308,00$ & $\mathrm{R} \$ 616,00$ & $\mathrm{R} \$ 1.153,09$ \\
\hline $\begin{array}{l}\text { Custo anual da Compra Descentralizada } \\
\text { (R\$) }\end{array}$ & $10.113 .339,60$ & $1.730 .617,92$ & $14.382 .147,36$ & $3.414 .124,80$ \\
\hline Custo anual da Compra Centralizada ( $\$$ ) & $5.696 .168,40$ & $857.472,00$ & $7.125 .888,00$ & $1.660 .449,60$ \\
\hline Economia pela Compra Centralizada $(\mathrm{R} \$)$ & $4.417 .171,20$ & $873.145,92$ & $7.256 .259,36$ & $1.753 .675,20$ \\
\hline Variação de preço (\%) & $-43,7$ & $-50,5$ & $-50,5$ & $-51,4$ \\
\hline
\end{tabular}

* Preços unitários informados pela SES/PE

** Percentagem de correção do período 03/2010 à 05/2012

Nota: $A D A=$ adalimumabe; $E T A=$ etanercepte; IFX = infliximabe. 
De acordo com a Figura 1, considerando o número de pacientes com AR em uso de anti-TNF-a, cadastrados no SISMEDEX $(n=525)$, os gastos mensais no estado de Pernambuco, em maio de 2012, considerando a compra descentralizada, chegariam a um valor estimado de $\mathrm{R} \$ 2.172 .007,24$, frente a $\mathrm{R} \$ 1.278 .331,50$ por meio da compra centralizada, perfazendo uma economia mensal de $41,15 \%$.

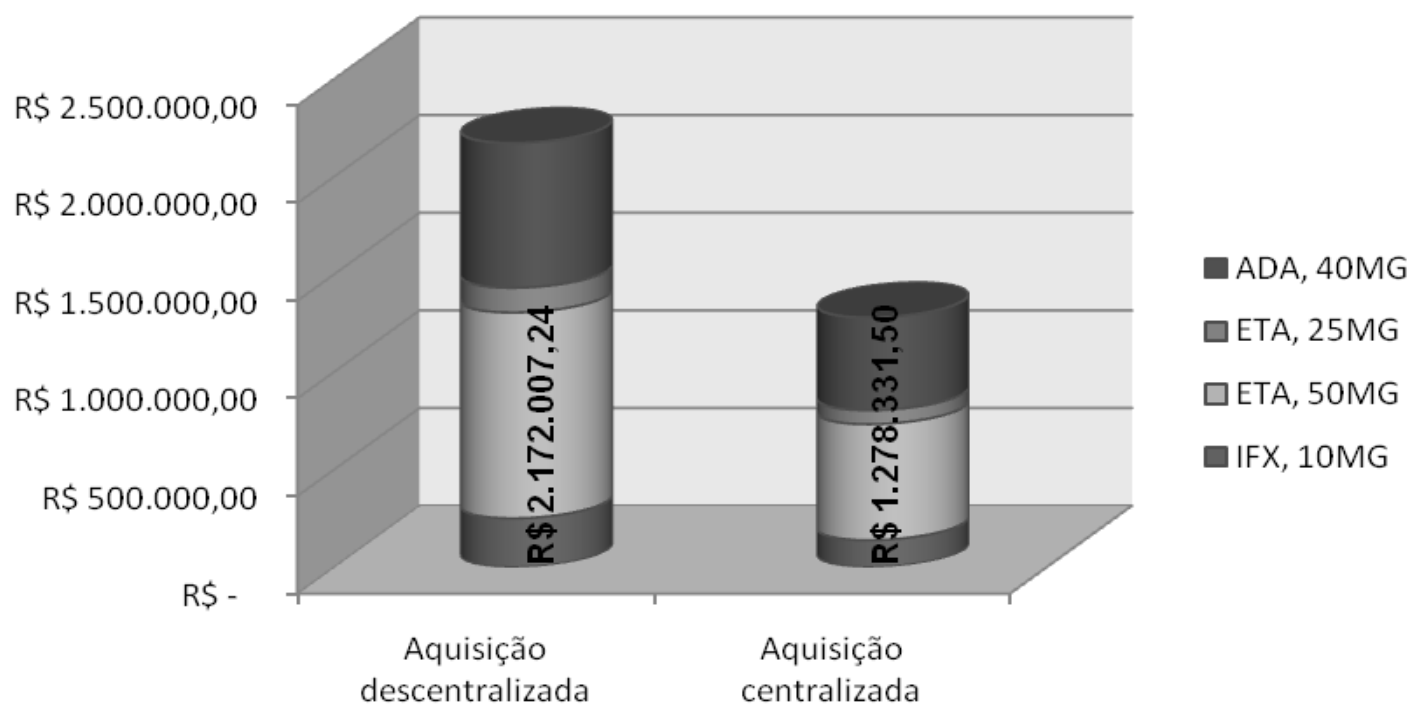

Fígura 1 - Comparativo do impacto mensal nos custos de aquisição dos anti-TNF-a entre a compra descentralizada versus a compra centralizada, no CEAF da SES/PE em maio de 2012.

\section{DISCUSSÃo}

O processo de compra centralizada pelo MS de anti-TNF-a para o tratamento da $A R$, evidenciou uma redução dos gastos com aquisição de medicamentos na SES/PE. Possibilitando desta forma uma maior economia aos cofres públicos, permitindo que, mais pessoas possam ter acesso ao tratamento e uma melhor realocação destes recursos economizados na saúde.
Estudo realizado por Do Amaral e Blatt (2011) demonstrou que a compra centralizada de medicamentos do componente básico através do Consórcio Intermunicipal de Saúde, além de ter possibilitado uma redução dos custos, houve uma redução na média de dias com falta de medicamentos e no número de itens em falta, causando um impacto positivo na Assistência Farmacêutica municipal e consequentemente na 
melhoria da qualidade de vida da população atendida ${ }^{20}$.

O impacto na redução dos gastos ocasionado pelo processo de compra centralizada do MS pode ser cogitado e explicado pelo fato do grande volume de medicamentos negociados e pela agilidade dinâmica no processo licitatório.

Considerando os limitados orçamentos públicos para a saúde, juntamente com os crescentes gastos para tratamento de doenças crônicas ocasionadas não apenas pelo envelhecimento populacional e transição epidemiológica, mas também pelo desenvolvimento $\mathrm{e}$ incorporação de novas tecnologias, é essencial que cada vez mais se incorpore novos modelos e conceitos de logística capazes de auxiliar na redução dos gastos na aquisição de medicamentos, permitindo que mais pessoas possam ter acesso aos tratamentos.

\section{CONCLUSÃO}

A aquisição centralizada dos anti-TNF-a para o tratamento da AR pelo MS possibilitou uma economia de recursos públicos na saúde em Pernambuco. Desta forma, o processo de compra centralizada pode ser uma importante ferramenta para a melhoria da gestão da Assistência Farmacêutica nos estados brasileiros. Por outro lado, a análise dos custos pela aquisição centralizada de antiTNF-a sugere que são necessários outros estudos que possibilitem fundamentar a análise de decisão para a compra centralizada de outros itens.

\section{AGRADECIMENTOS}

Agradecemos

à

Superintendência de Assistência Farmacêutica da Secretaria Estadual de Saúde de Pernambuco por ter possibilitado e apoiado à pesquisa, assim como a Comissão Permanente de Licitação de Medicamentos (CPLM) da SES/PE e a Universidade Federal de Pernambuco. E ainda, em especial, gostaríamos de agradecer a professora Suely Lins Galdino (in memória), pela sua orientação no início deste trabalho.

\section{REFERÊNCIAS:}

1) Brasil. Ministério da Saúde. Portaria no 3916 de 30 de outubro de 1998. Aprova a Política Nacional de Medicamentos. Diário Oficial da União, 1998.

2) Kondro W. Drug spending tops $\$ 25$ billion. Canadian Medical AssociationJournal, 2007;176(13): 1816. 
ZANGHELINE, F.; SILVA, A. S.; FILHO, J. A. R.

REF-ISSN1808-0804 Vol.XI (2),15-24, 2014.

3) Moynihan R.Drug spending in North America rose by $11 \%$ in 2003 . British Medical Journal, 2004; 328:7442-7727.

4) Vieira FS, Mendes ACR. 2007. Evolução dos gastos do Ministério da Saúde com medicamentos. [Acesso em 25 nov 2013]. Disponível em: http://portal.saude.gov.br/portal/arquivos/pdf/estudo gasto medicamentos.pdf.

5) Brasil. Ministério da Saúde. Portaria no 204, de 29 de janeiro de 2007. Regulamenta o financiamento e a transferência dos recursos federais para as ações e os serviços de saúde, na forma de blocos de financiamento, com o respectivo monitoramento e controle. Poder Executivo, Brasília.

6) Brasil. Ministério da Saúde. Portaria no 2.981, de 26 de novembro de 2009. Aprova o Componente Especializado da Assistência Farmacêutica. Poder Executivo, Brasília.

7) Vieira FS. Gasto do Ministério da Saúde com medicamentos: tendência dos programas de 2002 a 2007. Revista de Saúde Publica, 2009; 43(4):674-681.

8) Brasil. Ministério da Saúde. Portaria SCTIE no 710 de 27 de junho de 2013. Aprovar o Protocolo Clínico e Diretrizes Terapêuticas da Artrite Reumatóide. 2013

9) Conaghan PG, Green MJ, Emery P. Established rheumatoid arthritis. Bailliere'sBest Practice and Research in Clinical Rheumatology, 1999; 13(4): 561575.

10) Organizacao Mundial De Saude (OMS). [Acesso em 10mar 2014]. Disponível em: http://www.who.int/chp/topics/rheumatic/en/.

11) Englund $M$. Prevalence and incidence of rheumatoid arthritis in southern Sweden 2008 and their relation to prescribed biologics. Reumatology, 2010; 49:1563-1569. 
ZANGHELINE, F.; SILVA, A. S.; FILHO, J. A. R.

REF-ISSN1808-0804 Vol.XI (2),15-24, 2014.

12) Senna ER. Prevalence of rheumatic diseases in Brazil: a study using the COPCORD approach. The JournalofRheumatology, 2004; 31(3): 594-597.

13) Pincus $T$. The underestimated long term medical and economic consequences of rheumatoid arthritis. Drugs, 1995; 50(1):10-14.

14) Kojima M. Depression, inflammation, and pain in patients with rheumatoid arthritis. Arthritis Rheum, 2009; 61(8): 1018-1024.

15) Keystone EC. Radiographic, clinical, and functional outcomes of treatment with adalimumab (a human anti-tumor necrosis factor monoclonal antibody) in patients with active rheumatoid arthritis receiving concomitant methotrexate therapy: a randomized, placebo-controlled, 52-week trial. Arthritis and Rheumatism, 2004;50(5): 1400-1411.

16) Klareskog L. Therapeutic effect of the combination of etanercept and methotrexate compared with each treatment alone in patients with rheumatoid arthritis: doubleblindrandomised controlled trial. Lancet, 2004; 363(9410): 675681.

17) Maini R. Infliximab (chimeric anti-tumour necrosis factor alpha monoclonal antibody) versus placebo in rheumatoid arthritis patients receiving concomitant methotrexate: a randomised phase III trial. Lancet, 1999; 354(9194): 1932-1939.

18) Edwards JCW. Efficacy of B-cell-targeted therapy with rituximab in patients with rheumatoid arthritis. New England Journal of Medicine, 2004; 350(25): 25752581.

19) Genovese MC. Interleukin-6 receptor inhibition with tocilizumab reduces disease activity in rheumatoid arthritis with inadequate response to disease modifying antirheumatic drugs, Arthritis and Rheumatism, 2008;58(10): 29682980.

20) Do Amaral SMS, Blatt CR. Consórcio intermunicipal para a aquisição de medicamentos: impacto no desabastecimento e nos custos. Revista de Saúde Pública, 2011;45(4):709-801. 